\title{
Automatic Building Map Updating Using Worldview-2 Stereo Images and Existing Outdated Building Map
}

\author{
M. Gharibi *, H. Arefi , H. Rastiveis , H. Hashemi
}

Dept. of Surveying and Geomatics Eng, College of engineering, University of Tehran, Iran (mahdigharibi, hossein.arefi, hrasti, hosseinhashemi)@ut.ac.ir

KEY WORDS: Building Map Updating, Building Change Detection, Building Extraction, Active Contour Models

\begin{abstract}
:
Updating of existing geo-database, particularly in developing countries, is one of the important and essential issues in geospatial information systems. Numerous change detection methods have been proposed to resolve this issue. In this study, a novel approach is proposed for automatic building change detection and updating from available outdated building map and products of satellite stereo images. The proposed method consists of four steps. In the first step, preliminary building outline for a candidate building is extracted from available old polygon and DSM based on an active contour model. Then, some change detection rules are considered to find out whether or not the building has been changed. If changes are detected, the following third and fourth steps based on a hierarchical approach, run to generate precise changed building outline. The proposed method is tested and evaluated using sample dataset from Tunis City and the obtained results prove the feasibility of this algorithm for automatic building map updating using high resolution stereo satellite images.
\end{abstract}

\section{INTRODUCTION}

Map updating as one of the critical and important tasks in mapping agencies around the world is currently a manual process. Looking for an automated approach for detection and updating topographic objects such as buildings is an active research topic in academic institutions. Availability of new data and the use of appropriate algorithms for building extraction and change detection, are the most important success factors in building map updating process. The algorithm for building map updating usually consist of two main steps: building change detection and updating.

Based on available datasets in two different epochs, change detection methods can be classified into image-image and image-map comparison (Bouziani et al., 2010). In image-image strategy, two images, acquired in two different epochs compared based on common change detection techniques.

Image-map strategy uses an existing old map to detect changes between new data and existing geo-database. This strategy is more complicate than former because map data has a level of generalization and abstraction. This strategy is useful for feature extraction and change detection because it contains advantageous information about the relevant feature class.

Image-map change detection strategies are usually classified into post-extraction and map-guided methods (Bouziani et al., 2010). Post-extraction method is similar to post-classification methods that mentioned in image-image strategy. One of the difficulty of the post extraction method is deficiency of classification methods to extract features using classification techniques. Armenakis et al. (2003) has employed old maps of lakes for North of Canada as outdated map and Landsat 7 ETM+ images as newly dataset for lakes change detection. The change is defined as the non-intersection of the old and new vector features between two temporal spatial states.
In the map guided method, available information in the map is used to extract image objects from newly data. Existing outdated map database includes valuable information about object's location that facilitates the detection of interested objects. This method reduces the computational volume and errors in feature extraction from newly dataset. Bentabet et al. (2003) utilized existing map data to update road network. Bouziani et al. (2010) has used contextual information that is derived from map data and then has performed object-based classification using fuzzy rules to find changed buildings. Le Bris et al. (2011) has tested both image-map and image-image change detection strategy. In another study, Malpica et al. (2013) has used map-guided method for building change detection. He has used SVM classification technique for building extraction that training data are prepared from old map. In all aforementioned literature, one of the important steps in building map updating is building extraction process.

In numerous studies which has been done to detect the building changes in urban environment, small buildings which have area less than $50 \mathrm{~m}^{2}$ has been ignored in change detection process. These buildings are important in change detection process because many of changes are occurred in these buildings. In the present study a novel method is proposed for building change detection and updating that all building polygons has been utilized for building extraction and change detection processes.

\section{ACTIVE CONTOUR MODELS}

Several methods have been proposed to building extraction from photogrammetric and remote sensing data. Building extraction methods are usually classified into bottom-up (datadriven) and top-down (model-driven) approaches. In bottom-up approach, features are first detected, then constraints are progressively added, up to higher level recognition. Top-down approach defines what should be the object to search for, and

\footnotetext{
* Corresponding author.
} 
tries to localise it in the image (Liu and Prinet, 2005). Topdown approach have high robustness and less sensitivity to noise.

Among the building extraction methods, active contour models also known as snakes or moving fronts, usually are classified in top-down approach which firstly introduced by Kass et al. (1988). "A snake is an energy-minimizing spline guided by external constraint forces and influenced by image forces that pull it toward features such as lines and edges" (Kass et al., 1988). Nowadays, existing active contour models based on implementation method are classified into two main categories: parametric and geometric models. Parametric snakes are represented explicitly as parameterized contours, and the snake evolution is only performed on a predetermined spline control points (Ahmadi et al., 2010). Geometric models are represented implicitly as the zero-level sets of higher dimensional surfaces, and the updating is performed on the surface function within the entire image domain (Liu and Prinet, 2005). The basic idea of geometric models is to represent contours as the zero level set of an implicit function defined in a higher dimension, usually referred as the level set function, and to evolve the level set function according to a partial differential equation (PDE) ( $\mathrm{Li}$ et al., 2005).

Geometric active contours are introduced by Caselles et al. (1993) and Malladi et al. (1995). Geometric snakes have several advantages than parametric models (Sethian, 1999): First, the contours represented by the level set function can break or merge implicitly during the evolution, and therefore the topological changes are handled automatically. Second, the level set function always remains a function on an image that enables efficient numerical schemes. Geometric snakes, based on using information obtained from image, have been divided into two main classes: edge-based and region-based. Edgebased methods employ gradient information derived from image while, region based methods use homogeneity of pixel values at inside and outside of the contour to pull it to interesting image object boundary. Since in the present paper, both geometric snake types will be utilized, theoretical concepts of both methods are described in the next section.

\subsection{Theoretical background}

A region-based geometric active contour using Mumford-Shah function is proposed as an additional constraint to improve convergence range and flexibility in initialization that shown in following function (Chan and Vese, 2001):

$$
\begin{aligned}
& E(c)=\mu \text {.length }(c)+\text { v.area }(\text { inside }(c))+ \\
& \lambda_{1} \cdot \int_{\text {inside }(c)}\left|u-c_{\text {in }}\right|^{2} \mathrm{dxdy}+\lambda_{2} \cdot \int_{\text {outside }(c)}\left|u-c_{\text {out }}\right|^{2} \mathrm{dxdy}
\end{aligned}
$$

$\mathrm{C}, \mathrm{u}, \mathrm{C}_{\mathrm{in}}, \mathrm{C}_{\text {out, }}$ represent curve of snake, pixel value, average of pixel values inside and outside of curve, respectively. The $\mu, v$, $\lambda_{1}, \lambda_{2}$ are constants that $\mu, \lambda_{1}, \lambda_{2}$ should have positive value. The most important advantage of the proposed model is the less sensitivity to noisy images. Various works in related to this field have executed (Ahmadi et al., 2010; Lie et al., 2006).

As mentioned in previous section, edge-based geometric active contour is similar to region-based with difference that the edgebased methods utilize gradient information derived from image to move the contour towards object boundaries. Li et al. (2005) proposed new variational formulation for geometric active contours. They add a distance regularizing term to external energy function to improve stabilization of evolution. The proposed total energy function is defined as below: $\varepsilon(\phi)=\mu \rho(\phi)+\varepsilon_{g, \lambda, v}(\phi)$

where, the $\phi$ is level set function with three dimension. The above total energy function is defined by two terms:

$$
\rho(\phi)=\int_{\Omega} \frac{1}{2}(|\nabla \phi|-1)^{2} \mathrm{dxdy}
$$

and

$\varepsilon_{g, \lambda, v}=\lambda L_{g}(\phi)+v A_{g}(\phi)$

that, are internal and external energy function, respectively. In function evolution process, internal energy penalizes the deviation of function $\phi$ from signed distance function, while external energy function pull the active contour (zero level set) to image object boundary. External energy function has two terms:

$$
L_{g}(\phi)=\int_{\Omega} g \delta(\phi)|\nabla \phi| \mathrm{dxdy}
$$

and

$$
A_{g}(\phi)=\int_{\Omega} g H(-\phi)|\nabla \phi| \mathrm{dxdy}
$$

in which the equation 5 computes length of the zero level set, while the equation 6 cause to move faster the zero level set evolution. Here, $\delta$ and $\mathrm{H}$ are the Dirac and Heaviside function, respectively. $\mu, \lambda$ and $v$ are constants that $\mu, \lambda$ have positive value. The parameter $g$ in equation 5 and 6 is edge indicator:

$$
g=\frac{1}{1+\left|\nabla G_{\sigma} * I\right|^{2}}
$$

where "I" is input image and $\mathrm{G} \sigma$ is the Gaussian kernel with standard deviation $\sigma$. The following equation is the gradient flow that minimizes the functional $\varepsilon$

$$
\frac{\partial \phi}{\partial t}=-\frac{\partial \varepsilon}{\partial \phi}
$$

\section{PROPOSED METHOD}

The flowchart of the proposed method for building map updating is shown in Figure 2. The proposed method comprises four major steps, which are the building extraction in level 1 , building change detection, building extraction in levels 2 and 3 . Before the main processing, a pre-processing step is done. Each of these steps are explained in next sections.

\subsection{Data pre-processing}

A DSM is a digital surface model of the landscape that includes all above man-made and natural ground objects such as buildings and trees. The separation of buildings from nonterrain objects, particularly vegetation areas, is indispensable. This separation can be done using height roughness, vegetation mask generation or image classification. Moreover, shadow areas in stereo images make errors in image matching process and therefore lead to void areas in produced DSM. These areas are filled by a DSM filling procedure that cause to smoothing the DSM. Accordingly, to decrease the smoothing effect of the DSM as well as the removing the vegetation areas for building extraction process, a supervised pixel-based classification is carried out using available orthorectified multispectral image. The produced mask applied to DSM to enhance the buildings blob detection. Figure 1 shows the employed vegetation and shadow regions mask. 


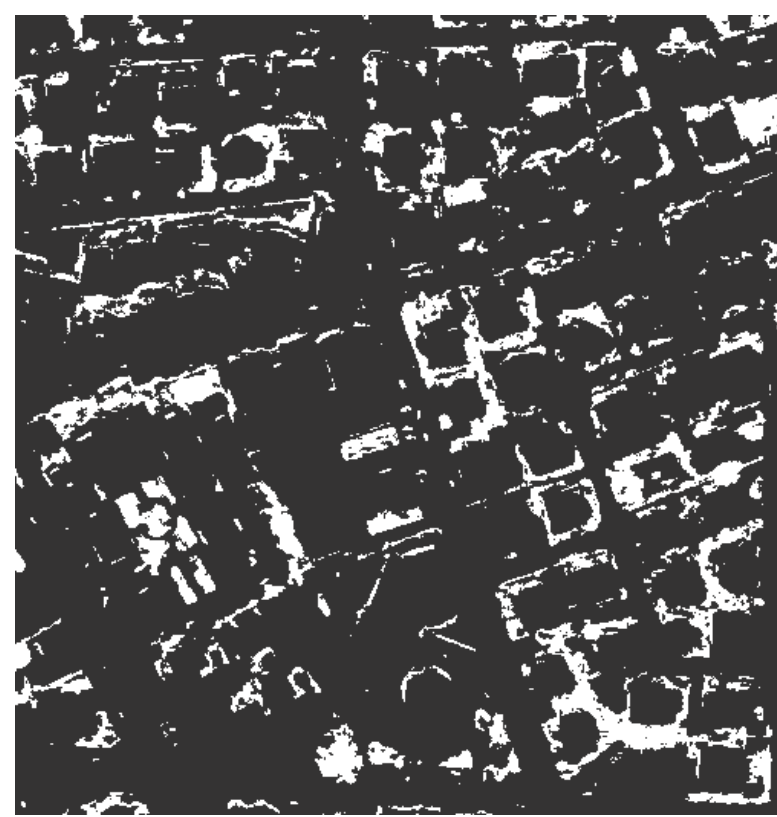

Figure 1. Vegetation and shadow areas mask derived from supervised classification of orthorectified multispectral image

\subsection{Main processing}

The flowchart of the proposed method for automatic building map updating using outdated building map, new DSM and orthorectified multispectral image from Worldview-2 satellite imagery is represented in Figure 2. To take advantages of existing outdated building map in building extraction and its updating, a map guided approach is utilized. As shown in Figure 2, after preprocessing phase, the proposed method continuous in four major steps: preliminary building extraction (level 1), building change detection, building extraction using PCA image (level 2) and building extraction using gray value image (level 3). In map guided approach for change detection, available outdated data is used for object detection and extraction from new dataset. For taking advantages of existing outdated building map in both building extraction and updating process, map guided approach is utilized.

In this study, available outdated building polygons are applied to preliminary building extraction from DSM. Each of the abovementioned steps will be described in oncoming paragraphs. The edge-based and region based active contour models have their pros and cons and selecting each of them in a specific application mostly depends on the purpose of the study and characteristics of the input images. Dissimilarity between elevation and spectral data on one hand and capability of region-based and edge-based geometric active contour models on the other hand caused considering both models during building extraction phase.

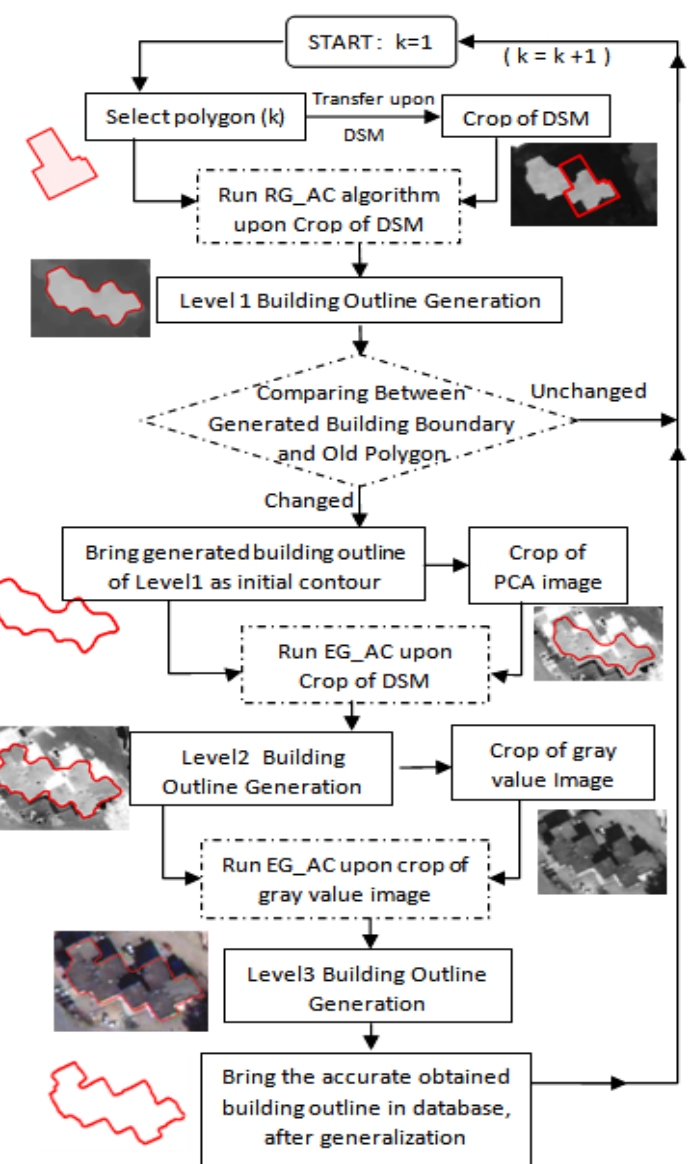

Figure 2. Flowchart of automatic building map updating

\subsection{Level 1 of building extraction process}

In this step, for each available outdated building polygons a bounding box with an acceptable buffer zone about 8 pixels, is considered on DSM. Although, derived DSMs from image matching techniques often do not produced sharp edges, they includes homogenous regions (blobs regions) upon non-terrain objects. For this reason, region-based geometric active contour (RG_AC) algorithm, introduced by chan et al. (2001), is used for preliminary building boundary extraction. In this step, this bounding box is considered for each of the available outdated building polygons and is placed on DSM. The selected polygon has been considered as initial contour for RG_AC algorithm and then it runs on DSM. The main specific of this algorithm is insensitivity to initial contour. So if the old building polygon has been changed, it could have no effect on result of RG_AC algorithm for building extraction over the DSM. Figure 3.(a) illustrates a sample of the changed building that is overlaid on DSM. The RG_AC handle this polygon as an initial contour and after running the algorithm, primary building boundary can be achieved (cf. Figure 3).

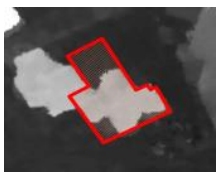

(a)

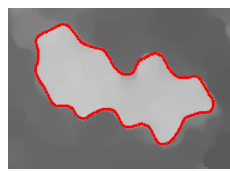

(b)
Figure 3. (a) Overlaid polygon and DSM, (b) Result of RG_AC 
In other words, the old polygon determines primary location of initial contour for RG_AC algorithm. The result of level 1 is preliminary outline of building that represents approximate building boundary. Figure 4 shows the results of RG_AC superimposed on the DSM.

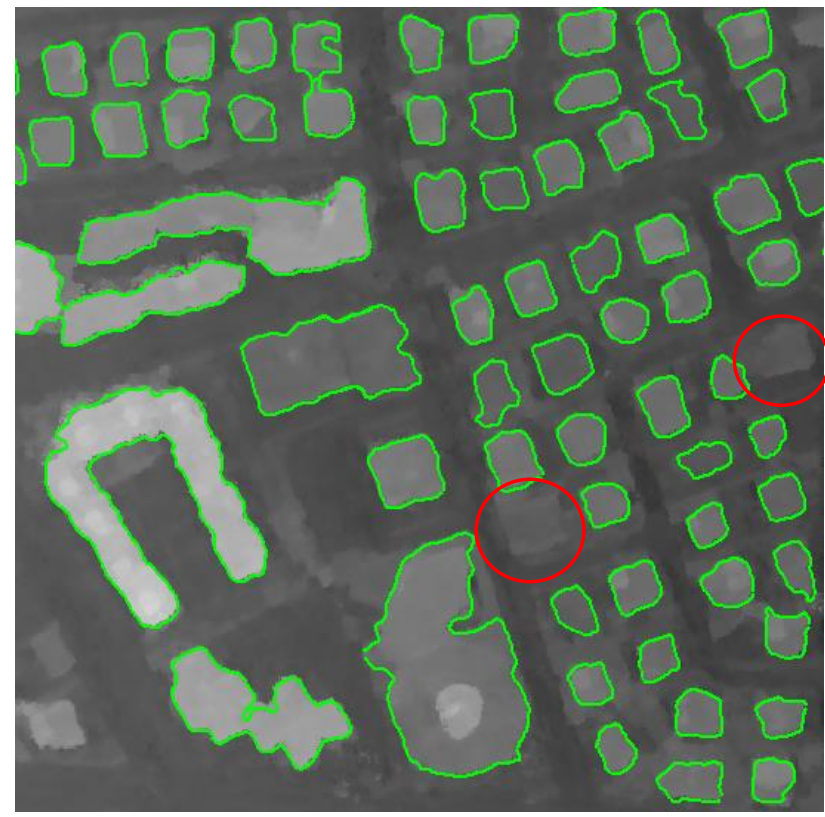

Figure 4. Results of RG_AC upon the DSM. The green boundaries are the output of level 1 in building extraction method and red regions are non-detected buildings.

\subsection{Building change detection}

After approximate building boundary production, comparison between outdated polygon and produced boundary is carried out in object based framework. Comparison between two objects is carried out using geometric criteria. For this purpose, the area and centroid criteria have been selected for comparison. If there is any meaningful difference between outdated polygon and produced approximate boundary produced in level 1 , current polygon is labelled as "Changed Building" and step 3 and 4 of above flowchart will be executed, otherwise, the polygon will be labelled as "Unchanged Building" and next polygon of outdated database will be evaluated.

One of the innovations of proposed method is that building extraction does not carried out completely for all of available building objects in outdated map. Accordingly this leads to reduction of computation time. In many building change detection studies such as (Matikainen et al., 2010; Zhu et al.; $\mathrm{ZN}, 2011$ ) the building extraction process is completely carried out in the first step and then, changes of buildings are determined.

\subsection{Level 2 of building extraction process}

For each labelled buildings as "changed" in building change detection step, level 2 and 3 of building extraction process are carried out. The goal of the level 2 is generation of more accurate building boundary than the level 1 . In this level, PCA image produced using 8 bands of orthorectified multispectral images of worldview-2, is utilized. Orthorectified multispectral images of available worldview-2 have been obtained by generated DSM data. Produced PCA image includes maximum information about building's rooftop and therefore, is suitable for feature extraction. For this purpose, firstly, a bounding box with a buffer zone about 8 pixel is created around the produced preliminary boundary in level 1 and is placed over PCA image. This boundary is then considered as an initial contour for edgebased geometric active contour (EG_AC) algorithm proposed by Li et al. (2005). Result of this process is an accurate building boundary rather than the output of level 1 (cf. Figure 5). Although, output of this step is admissible output, it needs to further processing to obtain better result since PCA image has not salient edges. Hence, third level of building extraction process will be executed.

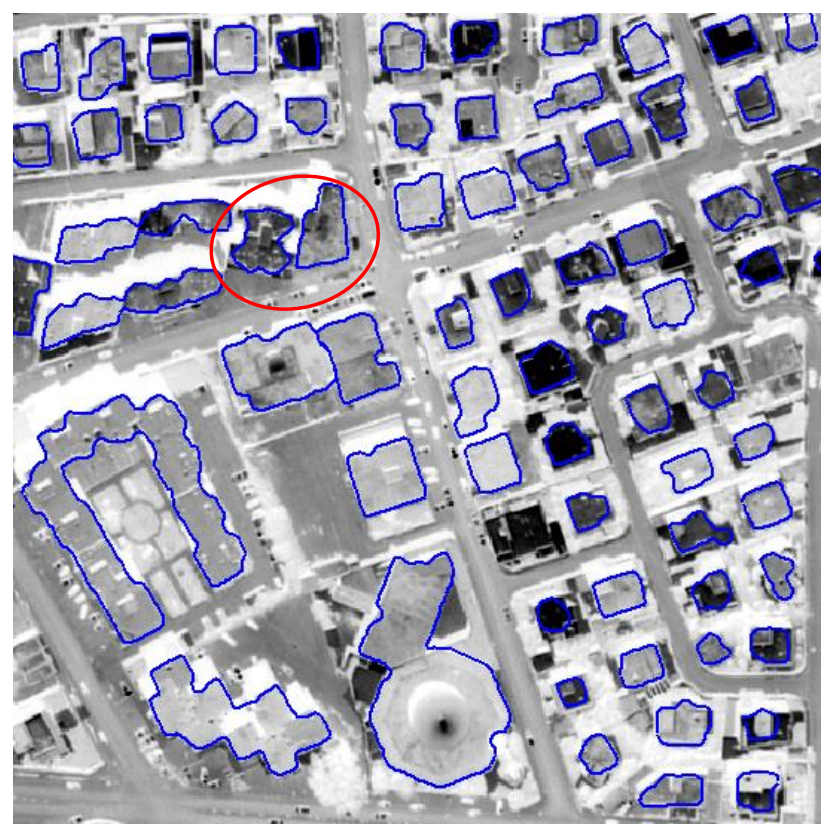

Figure 5. Results of EG_AC upon PCA image in level2 of building extraction. In the red region, the algorithm wrongly detects two distinct building instead of one building.

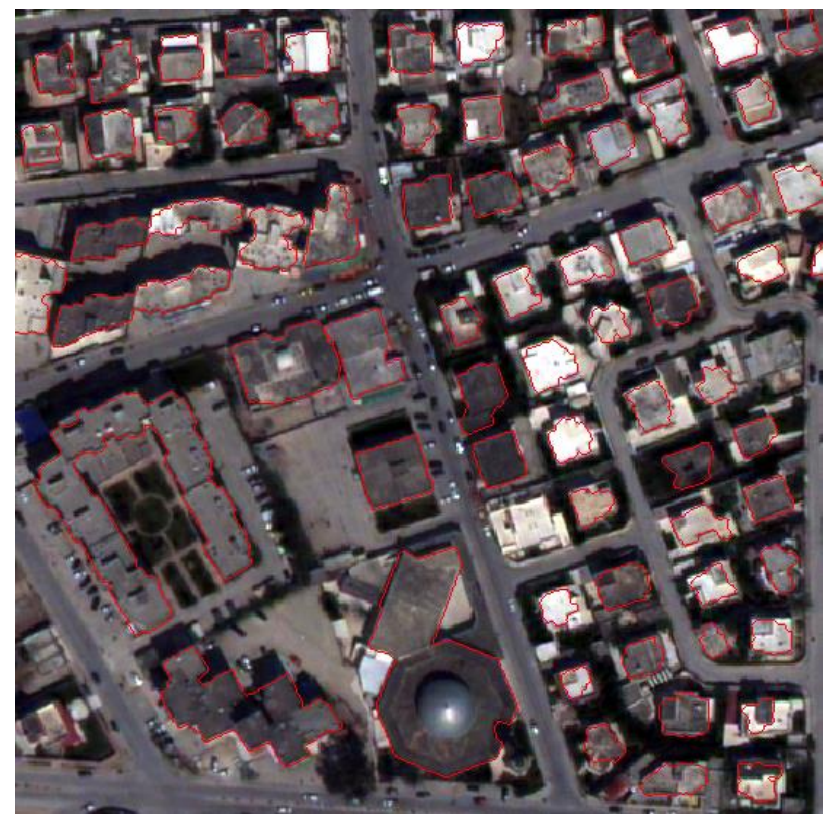

Figure 6. Results of EG_AC upon RGB image in level 3 of building extraction. Red boundaries are the output of level 3 . 


\subsection{Level 3 of building extraction process}

In this level, a grey value image obtained from fusion of channels 5, 3 and 2 of available pan-sharpened orthorectified image. The details of this image are better than PCA image and therefore, the snake can be coincide to a more accurate building boundary. For improve the results, dimensions of this image has been great 2 times the primary state which cause the snake to move towards corners of building, accurately. In this level alike two above levels, firstly, a bounding box is created around the boundary that generated in previous step (level 2) and is placed on grey value image. Then, the EG_AC algorithm with different parameters than previous level is executed. Figure 6 illustrates the final buildings outline generated in level 3 of building extraction method.

It can be clearly seen that the buildings outline in this level has better performance rather than boundaries produced by PCA image in level 2. In order to achieve better visual inspection, the reference building polygons has been superimposed on final boundaries which have been illustrated in Figure 7 .

\subsection{Detection of demolished and new buildings}

After executing RG_AC algorithm on DSM in level 1, if there is no corresponding image object in the new dataset for selected outdated building polygon, the polygon will be labelled as "demolished building".

For detection of new building, all of the changed buildings that have been extracted from new dataset and outdated building polygons are placed on DSM as mask regions. Then, RG_AC algorithm which has been used in level 1 for preliminary building extraction, is performed upon DSM. This process leads to segmentation of remaining areas and determination of building blobs. Afterwards, Levels 2 and 3 of building extraction process will be executed in order to obtain accurate building boundaries.

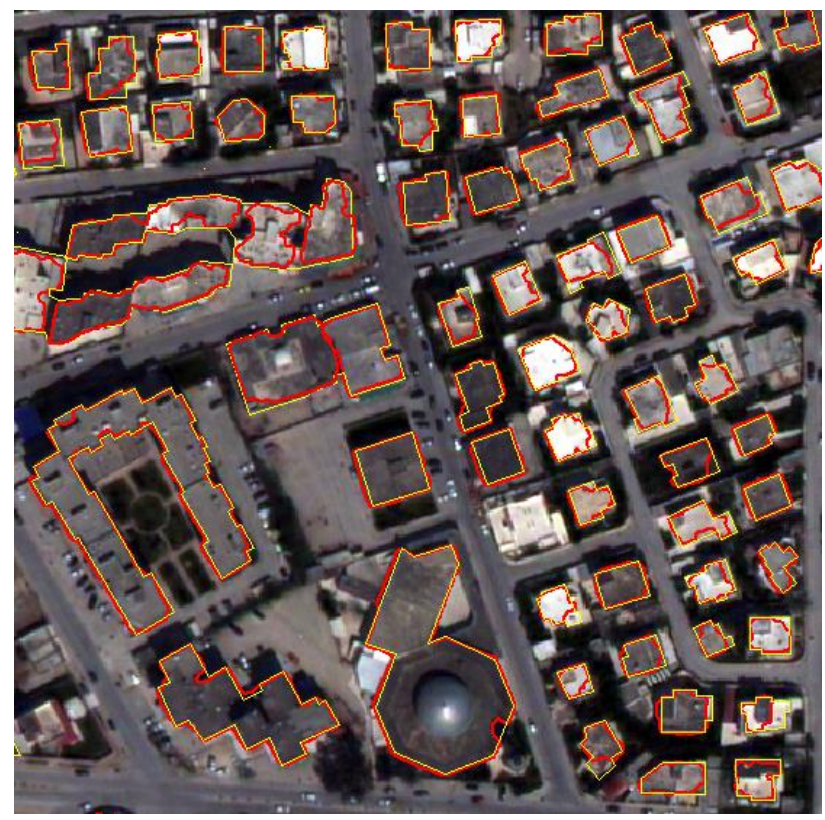

Figure 7. Overlaid reference polygons (yellow) and final results of map updating (red) over orthorectified worldview-2 satellite image

\section{RESULTS AND DISCUSSION}

The proposed algorithm for automatic building map updating using outdated buildings map, DSM and pan-sharpened orthorectified multispectral image from Worldview-2 satellite imagery has been tested in an area located at the Tunis, Capital of Tunisia. In order to achieve a quantitative evaluation for proposed method, the simulated outdated polygons is provided manually by adding, removing and changing buildings. Figure 8 shows simulated outdated map and reference data. As displayed in this Figure, there are 6 classes of building changes: shrinkage/enlarged, new, demolished, 1-n, n-1 and unchanged. The final results of proposed map updating method are shown in Figures 6 and 7. As shown in Figure 4, two buildings has not detected in the level 1 of building extraction method because the DSM in some of areas has low quality and in some areas, buildings have low height difference to the ground surface.

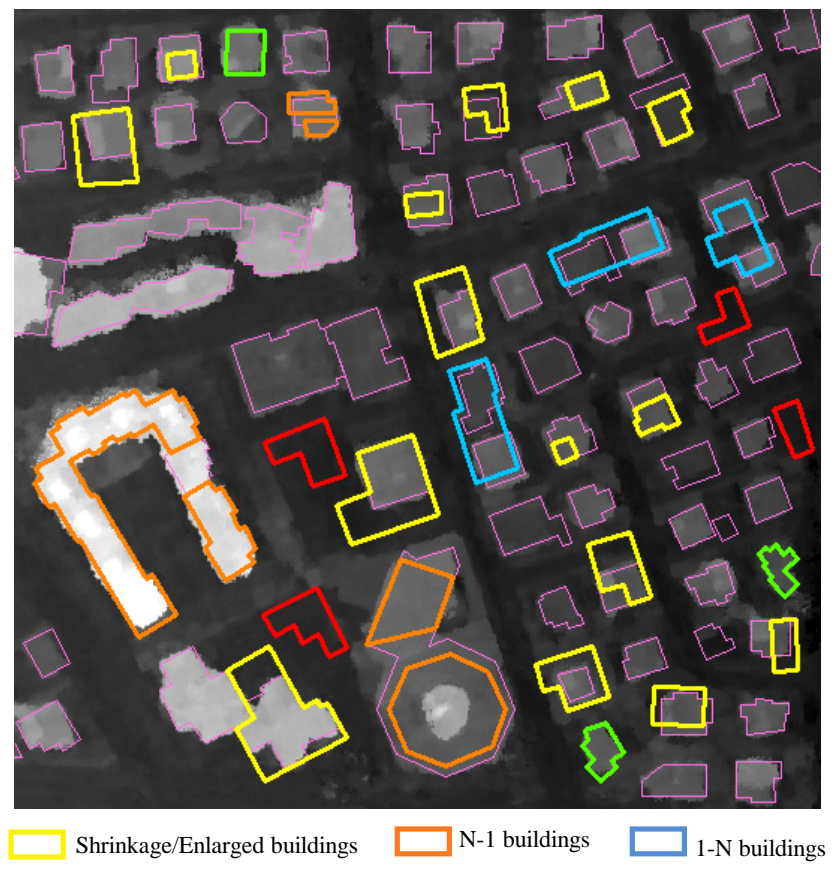

New buildings $\square$ Demolished buildings $\square$ Reference buildings

Figure 8. Simulated outdated map and reference building polygons upon the DSM.

Although geometric active contour models can handle topological changes which has been used in the present paper but this property isn't beneficial always. For example, in level 2 , some of big buildings with various rooftop textures have been wrongly decomposed to several buildings (cf. Figure 5). The proposed method for building map updating and building extraction relies on DSM data. So, if the DSM contains poor geometric quality, these two above process will not have acceptable performance. As mentioned in (Rottensteiner et al., 2005) the correctness and the completeness as 2 quality measures usually are used to assess the results of the change detection process:

$$
\begin{aligned}
& \text { Correctness }=\frac{T P}{T P+F N} \in[0 ; 1] \\
& \text { Completeness }=\frac{T P}{T P+F P} \in[0 ; 1]
\end{aligned}
$$

After performing the proposed building map updating method, the confusion matrix has been provided according to (Matikainen et al., 2010). The completeness and correctness measures have been obtained from confusion matrix. The values of these measures are $93 \%$ and $78 \%$, respectively.

Figure 9 represents the difference between area of reference polygons and corresponding extracted buildings outline in level 2 and 3 with blue and red lines, respectively. As shown in Figures 9, the difference between reference polygons and the 
corresponding extracted buildings outline in level 3 is less than level 2 which demonstrates the success of hierarchical approach for proposed building extraction method. The differences in level 2 are between $-28 \mathrm{~m}^{2}$ and $+58 \mathrm{~m}^{2}$ whereas, in level 3 , differences are between $-19 \mathrm{~m}^{2}$ and $+27 \mathrm{~m}^{2}$.

\begin{tabular}{|c|c|c|}
\hline Results Reference & $\begin{array}{c}\text { Changed } \\
\text { Buildings }\end{array}$ & $\begin{array}{c}\text { No Changed } \\
\text { Buildings }\end{array}$ \\
\hline Change Detection Results & $\begin{array}{c}\mathbf{3 0} \\
\text { (True detected) }\end{array}$ & $\begin{array}{c}\mathbf{8} \\
\text { (False alarm) }\end{array}$ \\
\hline No Changed Buildings & $\begin{array}{c}\mathbf{2} \\
\text { (Missed alarm) }\end{array}$ & $\mathbf{3 2}$ \\
\hline
\end{tabular}

Completeness $=93 \% \quad$ Correctness $=78 \%$

Table 1. Confusion matrix and quality measures

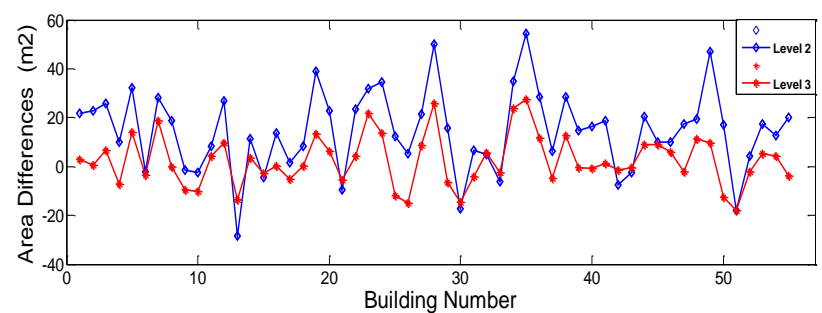

Figure 9. Difference between area of reference polygons and correspond correct extracted buildings outline in levels 2 and 3

\section{CONCLUSIONS AND FUTURE WORK}

An algorithm for automatic building map updating with aid of existing outdated building map was proposed based on mapguided approach in building change detection, utilizing a combination of active contour models in a hierarchical approach for building extraction process.

In this study, accurate building extraction has not been carried out for all buildings completely, but only for buildings that has been labeled "changed building" in change detection step. This leads to reduction of computation volumes, because this study had been considered that the changes between outdated map and new dataset has been derived from urban developments. One of the advantages of proposed method for building map updating is to take into account the available small building polygons $\left(\operatorname{area}<50 \mathrm{~m}^{2}\right)$ in change detection process, while in many change detection studies (Ioannidis et al., 2009; Le Bris and Chehata, 2011; Matikainen et al., 2010), these type of buildings are not considered and are removed in change detection step. For future work, a 3D map containing height information for building polygons can be used as outdated map database which leads to detect 3D changes of buildings that are still an interesting topic in this field.

\section{REFERENCES}

Ahmadi, S., Zoej, M., Ebadi, H., Moghaddam, H.A., Mohammadzadeh, A., 2010. Automatic urban building boundary extraction from high resolution aerial images using an innovative model of active contours. International Journal of Applied Earth Observation and Geoinformation 12, 150-157. Bouziani, M., Goïta, K., He, D.-C., 2010. Automatic change detection of buildings in urban environment from very high spatial resolution images using existing geodatabase and prior knowledge. ISPRS Journal of Photogrammetry and Remote Sensing 65, 143-153.

Chan, T.F., Vese, L.A., 2001. Active contours without edges. Image processing, IEEE transactions on 10, 266-277.
Ioannidis, C., Psaltis, C., Potsiou, C., 2009. Towards a strategy for control of suburban informal buildings through automatic change detection. Computers, environment and urban systems 33, 64-74.

Kass, M., Witkin, A., Terzopoulos, D., 1988. Snakes: Active contour models. International journal of computer vision 1, 321-331.

Le Bris, A., Chehata, N., 2011. Change detection in a topographic building database using submetric satellite images. International Archives of the Photogrammetry, Remote Sensing and Spatial Information Sciences 38, 3.

Li, C., Xu, C., Gui, C., Fox, M.D., 2005. Level set evolution without re-initialization: a new variational formulation, Computer Vision and Pattern Recognition, 2005. CVPR 2005. IEEE Computer Society Conference on. IEEE, pp. 430-436.

Lie, J., Lysaker, M., Tai, X.-C., 2006. A binary level set model and some applications to Mumford-Shah image segmentation. Image Processing, IEEE Transactions on 15, 1171-1181.

Liu, W., Prinet, V., 2005. Building detection from highresolution satellite image using probability model, INTERNATIONAL GEOSCIENCE AND REMOTE SENSING SYMPOSIUM, p. 3888.

Matikainen, L., Hyyppä, J., Ahokas, E., Markelin, L., Kaartinen, H., 2010. Automatic detection of buildings and changes in buildings for updating of maps. Remote Sensing 2, 1217-1248.

Rottensteiner, F., Trinder, J., Clode, S., Kubik, K., 2005. Using the Dempster-Shafer method for the fusion of LIDAR data and multi-spectral images for building detection. Information Fusion 6, 283-300.

Rutzinger, M., Rüf, B., Vetter, M., Höfle, B., 2010. Change detection of building footprints from airborne laser scanning acquired in short time intervals. na.

Zhu, L., Shimamura, H., Tachibana, K., Li, Y., Gong, P., Building change detection based on object extraction in dense urban areas. International Archives of Photogrammetry, Remote Sensing and Spatial Information Science, Beijing, China 37, 905-908.

ZN, I., 2011. Automatic extraction and building change detection from digital surface model and multispectral orthophoto. Geodetski vestnik 55, 1 .

Armenakis, C., et al. (2003). "A comparative analysis of scanned maps and imagery for mapping applications." ISPRS Journal of Photogrammetry and Remote Sensing 57(5): 304314.

Bentabet, L., et al. (2003). "Road vectors update using SAR imagery: A snake-based method." Geoscience and Remote Sensing, IEEE Transactions on 41(8): 1785-1803.

Caselles, V., et al. (1993). "A geometric model for active contours in image processing." Numerische mathematik 66(1): $1-31$.

Malladi, R., et al. (1995). "Shape modeling with front propagation: A level set approach." Pattern Analysis and Machine Intelligence, IEEE Transactions on 17(2): 158-175.

Malpica, J. A., et al. (2013). "Change detection of buildings from satellite imagery and lidar data." International Journal of Remote Sensing 34(5): 1652-1675. 